\title{
Size Survey - Process chain and available products
}

\author{
Anton PREISS, Ulrich BOTZENHARDT* \\ Human Solutions $\mathrm{GmbH}$, Kaiserslautern, Germany
}

\begin{abstract}
Nowadays, size surveys is a point of discussion, planning and realisation in many countries. They can be also a topic for companies as well for armies. Traditionally size surveys were mainly conducted to acquire body measurements of the population. Since 3D body scanning is available the potential on size surveys have been remarkably raised. The automatically generated statistical analysis is now based on the pre-filtered information of the population, optimisation of garment's body measurement charts, individual and statistical avatars, physical mannequins and so on.

Taking size surveys is much more than just acquiring body measurements. Today a certain amount of demographic information is stored per person to filter for specific measurement tables. Body dimensions are taken by many people in parallel based on body scans acquired at different locations. Individual analysis should be provided for the big variety of interested customers such as armies, companies and all others that can take benefit of it. To keep all the customers and the people taking the survey satisfied, an efficient infrastructure and a certain amount of specialised tools is necessary. Due to size surveys such as SizeGERMANY, a good existing infrastructure and helpful tools are already available and ready for action right now.
\end{abstract}

Keywords: size survey, 3D body scanning, scanatar, body measurement chart

\section{Introduction}

Size surveys conducted using 3D body scanning is the beginning of a new way of acquiring body measurements from human beings. Before getting started with the scanning of population it is necessary to define what body dimensions should be taken regarding what the end results of the size survey are intended for. Once the definitions of body dimensions based on standards like ISO 7250 [1] and ISO 8559 [2] are made, a number of various scanning postures per person can be derived.

A sample should be taken while not all individuals of the population of interest can be scanned. The sample size depends on the variety of attributes such as gender, age group, regional adherence etc. as well as the accuracy of the measurement results.

When using 3D body scanners it should be defined where to store and how to backup the acquired data as well as how to make results available. One of the next steps is the setup of the infrastructure on how 3D body scans are being handled and creation of questionnaire. When setting up such an infrastructure the possibility of post processing of acquired data should be thoroughly thought of as e.g. the measurement extractions can be done by multiple operators at different places e.g. headquarter.

After the measurement extraction is complete it can be proceeded with the analysis of the extracted body dimensions. Standard body measurement charts can be defined and published. By means of using an internet based platform results can be made available to public.

Surveys based on 3D body scans offer additional possibilities and values. In comparison to the avatars which get generated based on two-dimensional measurements and are artificial, scanatars are generated based on one (individual scanatar) or many scans (statistical scanatar) of individuals. Availability of scanatars opens the door to the world of the virtual reality. Scanatars can be used in applications like vidya (garmet simulation) or Ramsis (ergonomic simulation in the automotive industry) and also for the production of physical mannequins e.g. to give production site the possibility to validate the fit of the produced garment.

\section{Getting started with the survey Benefits}

Nowadays commercial companies as well as armies are interested to know more about populations. As an example in the apparel industry it is essential to know whether the population behind the target group fits the garment. Once there is a detailed knowledge regarding the body dimensions, garment sizes which cover more people of the population than before can be developed. Size ranges can be adopted as well. Quite often garment sizes are offered where no buyers are and the majority of the buyers are forced to make a selection out of a few suitable garment sizes.

\footnotetext{
*ulrich.botzenhardt@human-solutions.com
} 


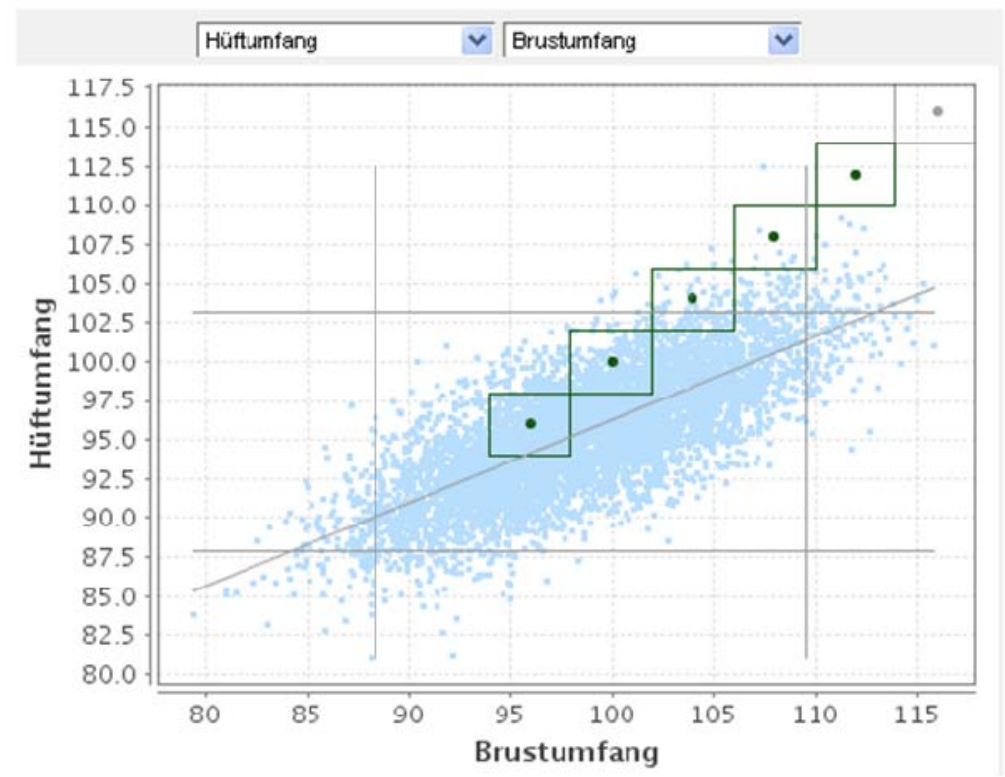

Fig. 1. Distribution of subjects regarding hip and chest and body measurement chart.

Companies that supply their employees with work wear are interested in good distribution of sizes. Based on the knowledge of body dimensions the quantity of sizes in stock can be optimized.

Simulation of garment based on statistical scanatars opens new possibilities for the pattern designer in the apparel industry. Newly designed patterns can be simulated immediately on scanatars either in the basic size or in graded sizes.

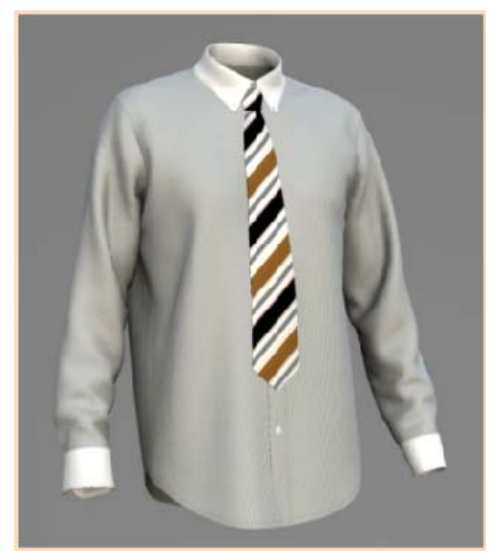

Fig. 2. Simulation of a shirt on a scanatar.

The knowledge of body dimensions can be of a big interest also for ergonomics. Car manufacturers for example are interested in body dimensions of the customers from different countries. Here the field of investigation is the reachability of the switches, sufficiency of the seat adjustment, availability of the headroom and so on.

\section{Population}

Each country has its individual population and attributes. The aim of the size survey is to get the actual population data. To receive a representative survey output it is highly important to measure a large number of randomly-selected representatives of each group of the population. The primary requirement for such serial measurement is that it enhances the entire population.

Important decision is how to make selection of participants to be measured. Several criteria influence this decision:

- gender

- age groups

- regional variations

- socio-demographic structure 
The number of age groups may wary according to the request of the survey. So, it can be the whole population or a particular age group. To receive good regional variations results representatives of all regions of the country should be selected.

One more important aspect is what sample size per group should be taken. When sample size per group is determined the minimum requirement for test persons can be calculated: as an example $10 \mathrm{~mm}$ for body length and $95 \%$ confidence, this will result in a sample size of at least 165 persons per group (Fig. 3).

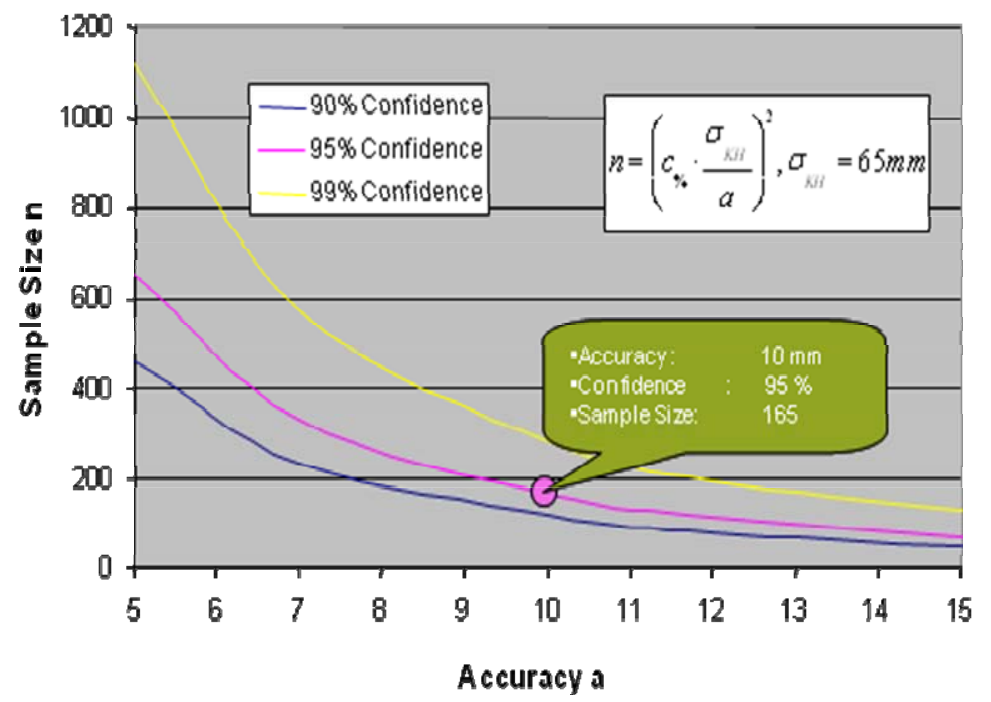

Fig. 3. Sample size per group based on confidence interval.

When sample size per group is determined the minimum requirement of test persons can be calculated:

number of subjects $=$ group size ${ }^{*}$ number of age groups ${ }^{*}$ number of regions

\section{Age groups}

\begin{tabular}{|c|c|}
\hline Population & Age Groups \\
\hline \multirow{2}{*}{ Girl } & $6-10$ years \\
& $11-13$ years \\
& $14-17$ years \\
\hline \multirow{2}{*}{ Boy } & $6-10$ years \\
& $11-13$ years \\
& $14-17$ years \\
\hline \multirow{3}{*}{ Women } & $18-25$ years \\
& $26-35$ years \\
& $36-45$ years \\
& $46-55$ years \\
& $56-65$ years \\
& Over 65 years \\
\hline \multirow{2}{*}{ Men } & $18-25$ years \\
& $26-35$ years \\
& $36-45$ years \\
& $46-55$ years \\
& $56-65$ years \\
& Over 65 years \\
\hline
\end{tabular}

Fig. 4. Typical age groups. 


\section{Confidence level}

For a $95 \%$ confidence level (which means that there is only a $5 \%$ chance of your sample results differing from the true population average), a good estimate of the margin of error (or confidence interval) is given by $1 / \sqrt{ } \mathrm{N}$, where $\mathrm{N}$ is the number of participants or sample size [4].

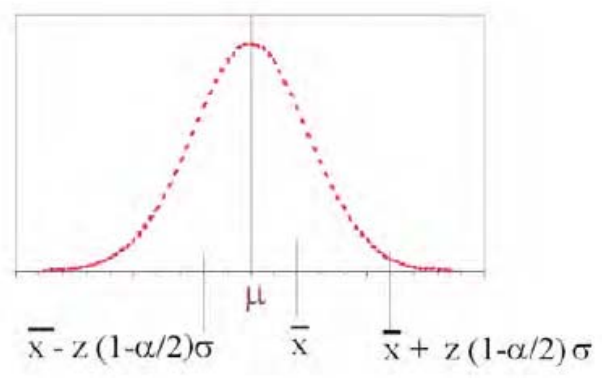

Fig. 5. Confidence level.

\section{Personal and demographic data}

Per each individual of the size survey the body scans and socio-demographic information is collected. To be sure that the sample is representative the socio-demographic data can be compared to the data of federal office of statistics. Additionally, information is needed to filter the population regarding the requirement of the target group per interest.

Typical socio-demographic data:

- Gender

- Age

- Family status and composition

- Nationality

- Religion

- Household size and own children

- Education

- Employment

- Job (classified according ISCO 88) and position

- etc.

\section{Posture and Body dimensions}

The decision how many postures should be used to carry out a survey and what measurements should be taken depends on the purpose of the survey and if the ISO standards should be taken into account. Thus the suitable postures for carrying out a study with body scanners are specified by ISO 20685 which suggests four postures - three standing and one sitting. The ISO 20685 also includes different size definitions in the ISO 7250 and ISO 8559 and recommends the necessary data sets. As for the selection of body dimensions, it is possible to compile a set according to the requirements of the survey. It is advantageous to use here ISO standards as well as the results will already correspond to the way the body dimensions are used for the development of the products. The results can be also easily compared with those of the older surveys taken based on the same standards. For the sizeGERMANY project both ISO Standards ISO 7250 and ISO 8559 were rigorously adhered to. In cooperation with the industry partners, 43 body dimensions were defined for the apparel industry [2] and 37 body dimensions for technical ergonomics [1]. 15 special body dimensions were also defined (used in the creation of 3D human models (e.g. RAMSIS) based on the measurements [5]. 


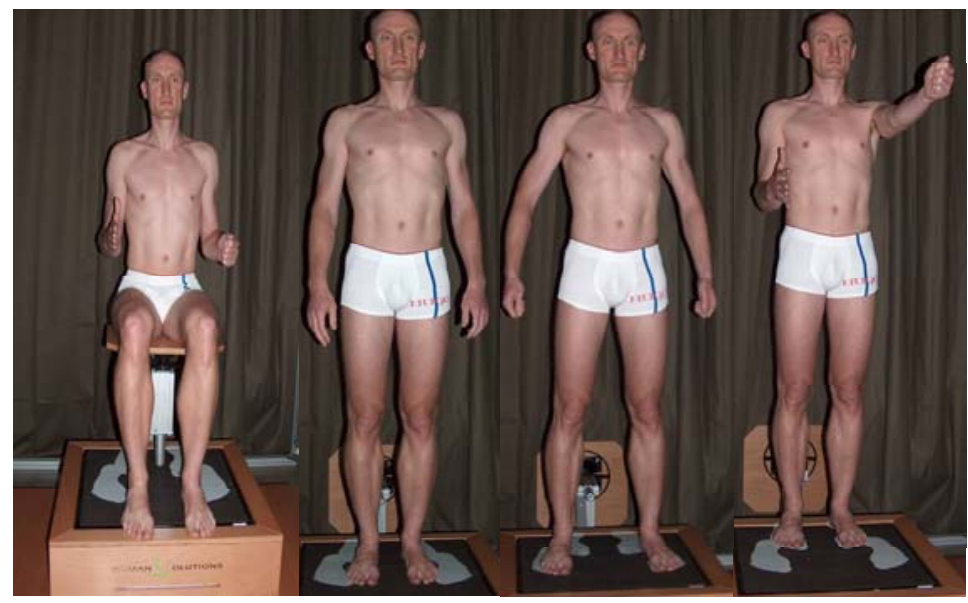

Fig. 6. Different scanning postures per subject.

Four postures (three standing postures and one sitting posture) were defined for the analysis of 95 body dimensions. On the right in Fig. 6 , the standing posture is represented by a stretched and angled arm posture - this enables the measurement of functional dimensions in standing posture. The second posture from the right (Fig. 6) is a specific measurement posture for body circumferences. The third measurement posture from the right in Fig. 6 is a relaxed standing posture to determine the correct body length. The posture on the left in Fig. 6 shows the sitting posture with bent arms in order to correctly acquire all necessary measurements in sitting posture. In addition, the weight of test persons can be determined electronically during scanning procedure. There is also an option to include an electronic questionnaire for the collection of socio-demographic information.

\section{Data acquisition}

Subjects of a survey are acquired at different locations with respect to the number of regions, age groups and gender.

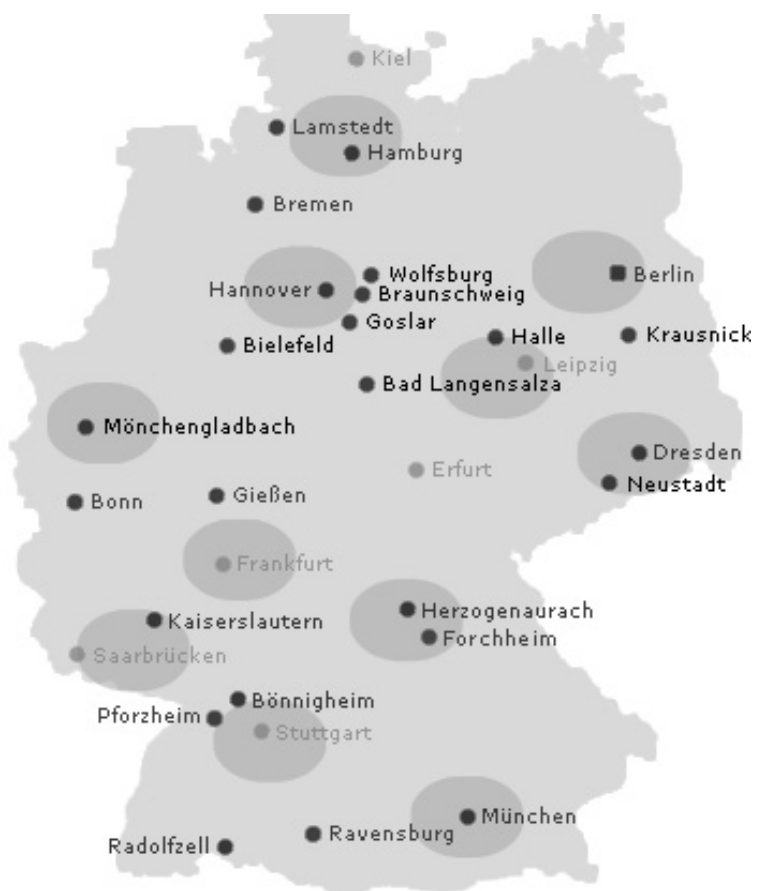

Fig. 7. Scanning locations of the German size survey sizeGERMANY.

Each survey participant has to be scanned in different postures and fill out a questionnaire.

The postures are defined by the previously selected body dimensions. In the German size survey sizeGERMANY the subject was scanned in four different postures, three standing and one sitting posture. Furthermore, more than one hundred questions were answered by each participant by means of a web based questionnaire. 


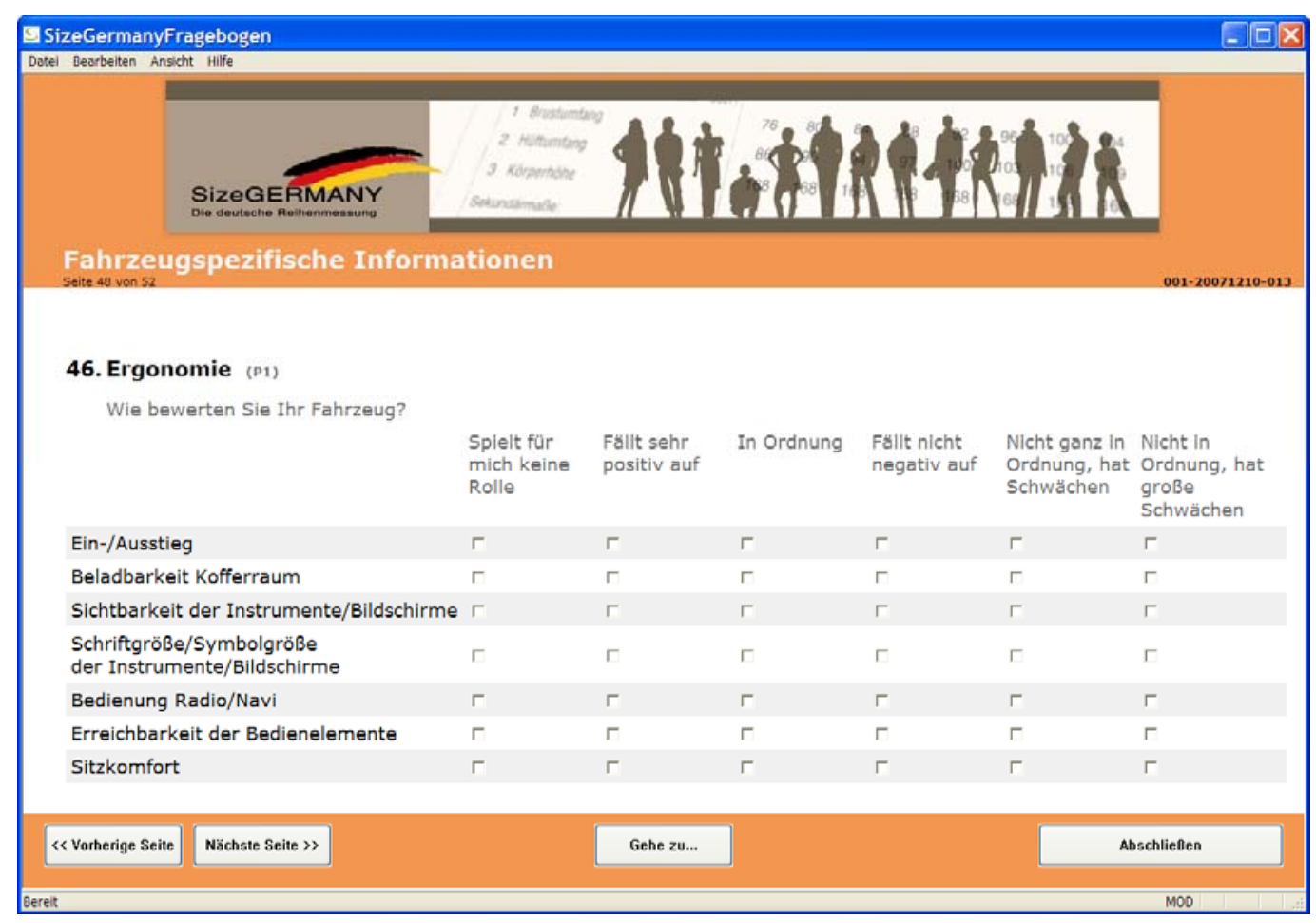

Fig. 8. Web based questionnaire used in the German size survey sizeGERMANY.

Each group should contain the necessary amount of subjects, not less, not more. A special functionality is needed to visualize missing subjects per group and groups with already enough subjects. Fig. 9 shows the example of distribution of female subjects over four different regions and nine age groups. Region "Süd" in age groups "18-25", "26-35", "36-45", "46-55", " $56-65$ " and "65+" is already filled, age groups "6-10", "11-13" and "14-17" still need some additional subjects. In all other regions in all age groups subject are needed.

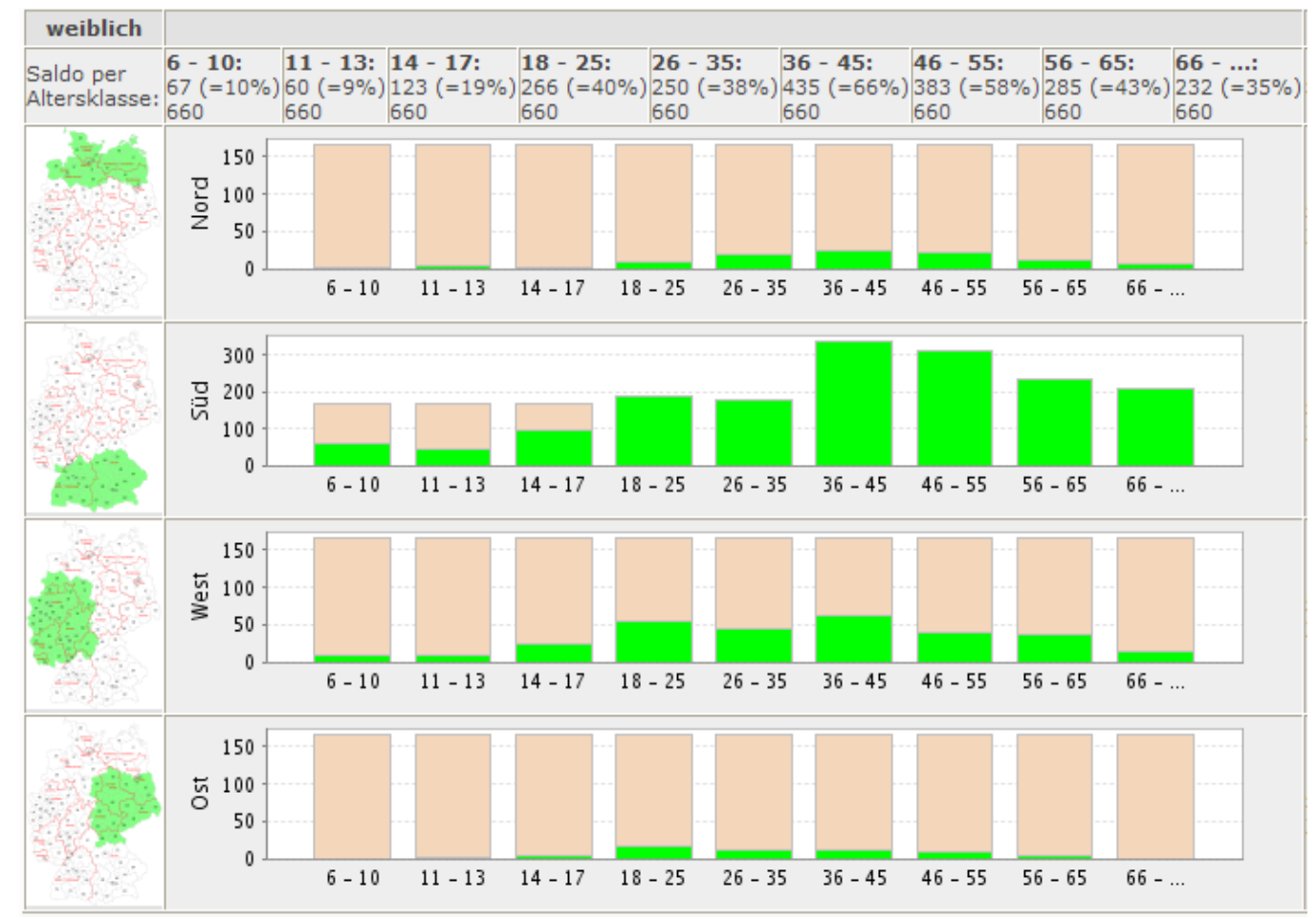

Fig. 9. Distribution of female subjects over four regions and 9 age groups. 
For storing of scanned data in a central database acquired from different locations as well as from parallel working scanning groups and controlling of the number of subjects per group a powerful tool is necessary. Anthroscan Scan Server, a product from Human Solutions, offers both functionalities.

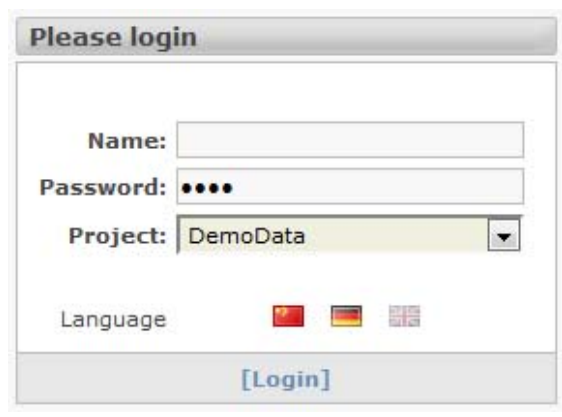

Fig. 10. Anthroscan Scan Server - Login.

Once scan data of a subject is available the body dimensions have to be extracted from the scans. Every single body dimension is defined by a specific scan posture that is why an assistant is necessary to support the operators taking all body dimensions.
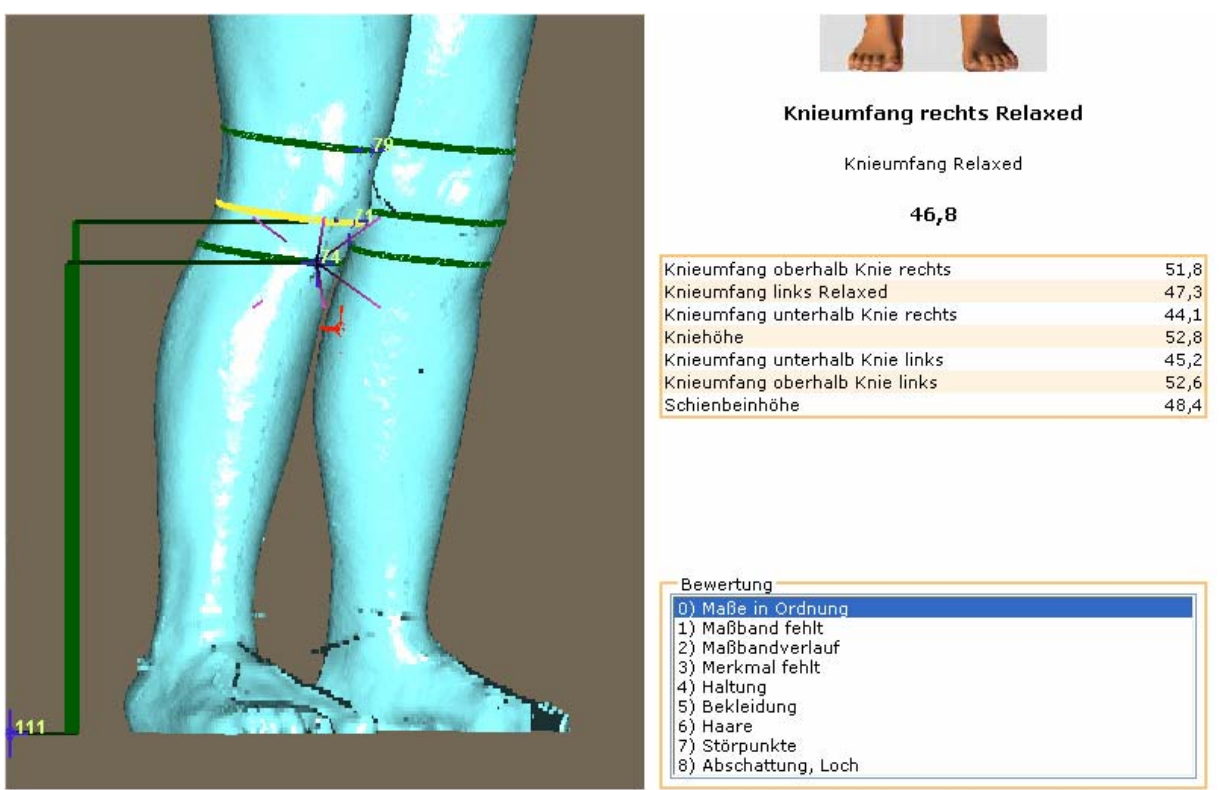

Fig. 11. Visualization of a body dimension including the assessment with related measurements.

Anthroscan Evaluation Assistant was used in the German size survey to support parallel working operators. The task of the operator was to define or modify preset landmarks on each scanned subject at different postures. Based on the landmarks body measurements were calculated. Every body measurement was checked by the operator and assessed.

The data quality is managed in a process orientated way, see Fig. 12:

1. Data acquisition

- Subject is scanned in 4 different postures

- Electronic questionnaire is completed

- Local storage of data

2. Data transfer to central database

- Check of data integrity, scanner quality and system calibration

- Monitoring of sample distribution

3. Initial automatic extraction of body dimensions

4. Data transfer to central database

5. Transfer of initial measurements to local database

6. Interactive inspection of measurements by specialists

7. Transfer of approved measurements in local database

8. Transfer of final measurements in central database 


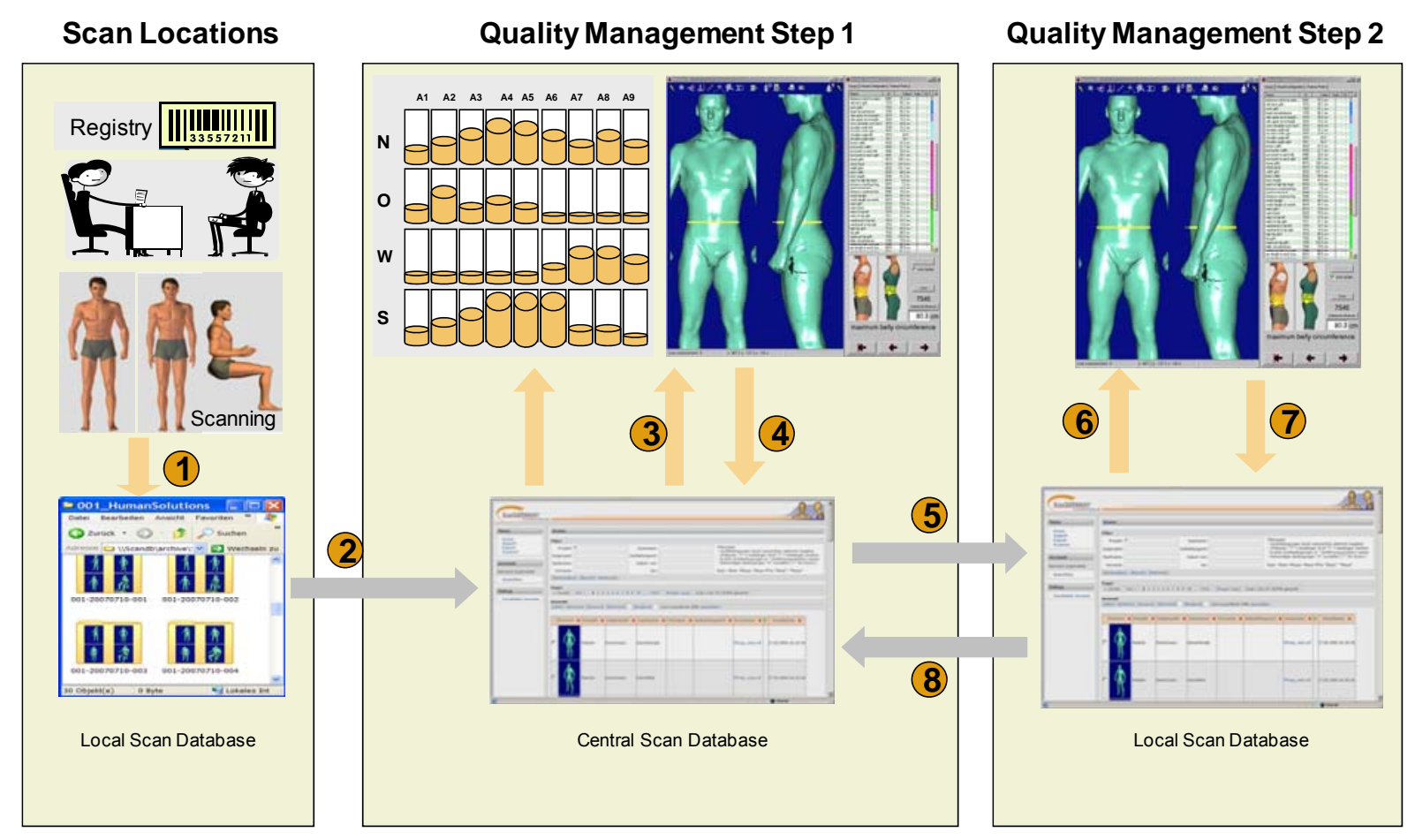

Fig. 12. Process and data flow per subject.

\section{Results and conclusion}

Analysis regarding changes through years and ages can be conducted after the extraction of body dimensions from all subjects of the sample is done and integrated into the database. The results of a survey can be compared to documented values from the German standard DIN 33402 [3]. In the German size survey sizeGERMANY the 50 percentile provides larger values than documented in the standard and the difference varies depending on the subject's age. In addition a comparison of extreme values (5. and 95. percentile) to documented values brings new knowledge regarding the changes of the population.

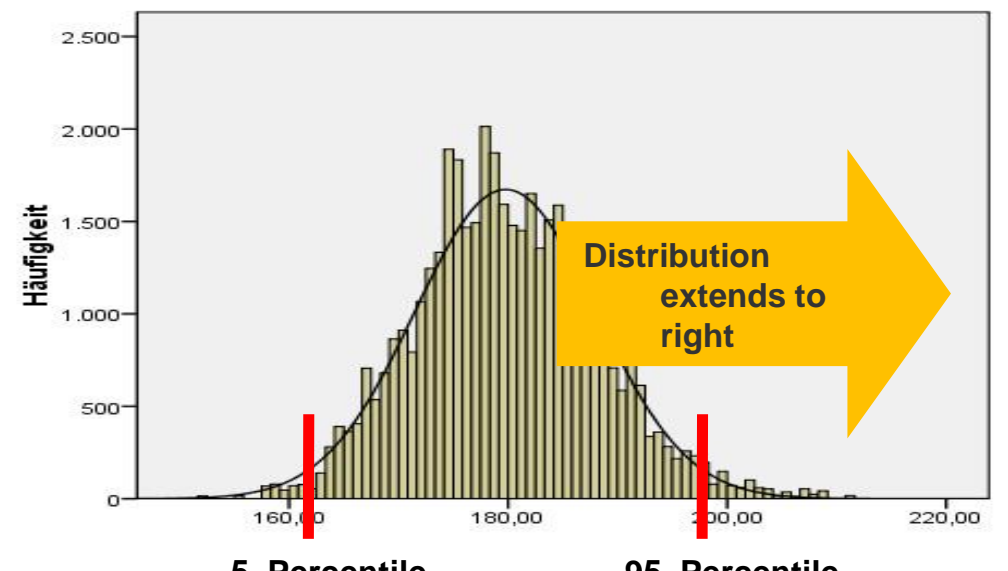

5. Percentile

95. Percentile

Fig. 13. Distribution of body height (male) from German size survey sizeGERMANY. 


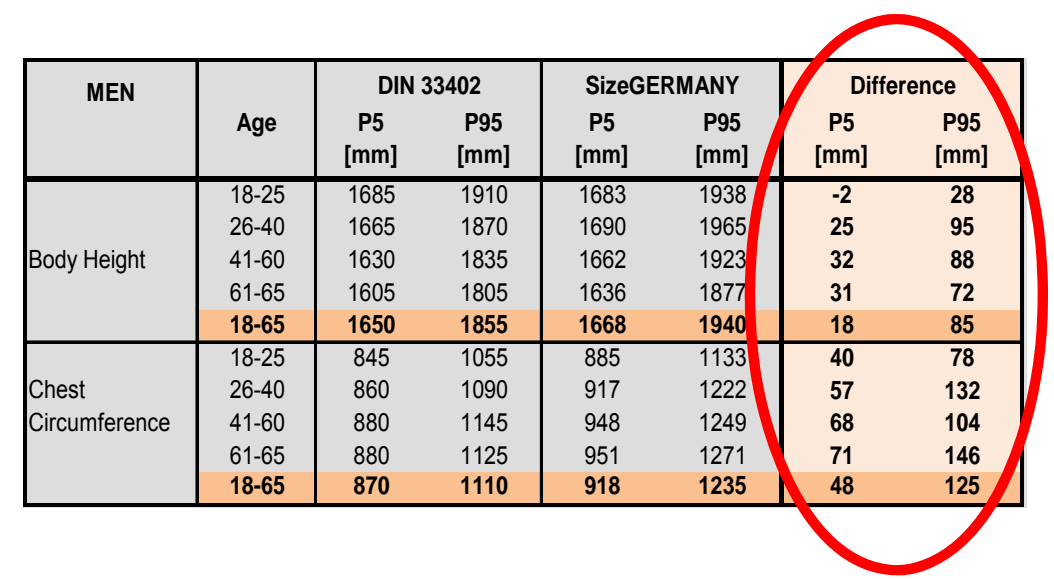

Fig. 14. Body height and chest circumference comparison of German's size survey sizeGERMANY with standard DIN 33402.

While individual data analysis is requested, it is necessary to filter the represented population. Such filter mechanism needs to cover such basic attributes like gender, regional adherence, age group as well as socio-demographic attributes and body dimensions. Analysis functions (distribution, average, deviation, percentiles, correlations \& regressions), optimization of market share tables, analyzed socio-demographic data and body dimensions can be used with the filter mechanism.

The web-based solution iSize from Human Solutions covers the described requests plus additional possibilities like calculation of body measurement tables and statistical scanatars.
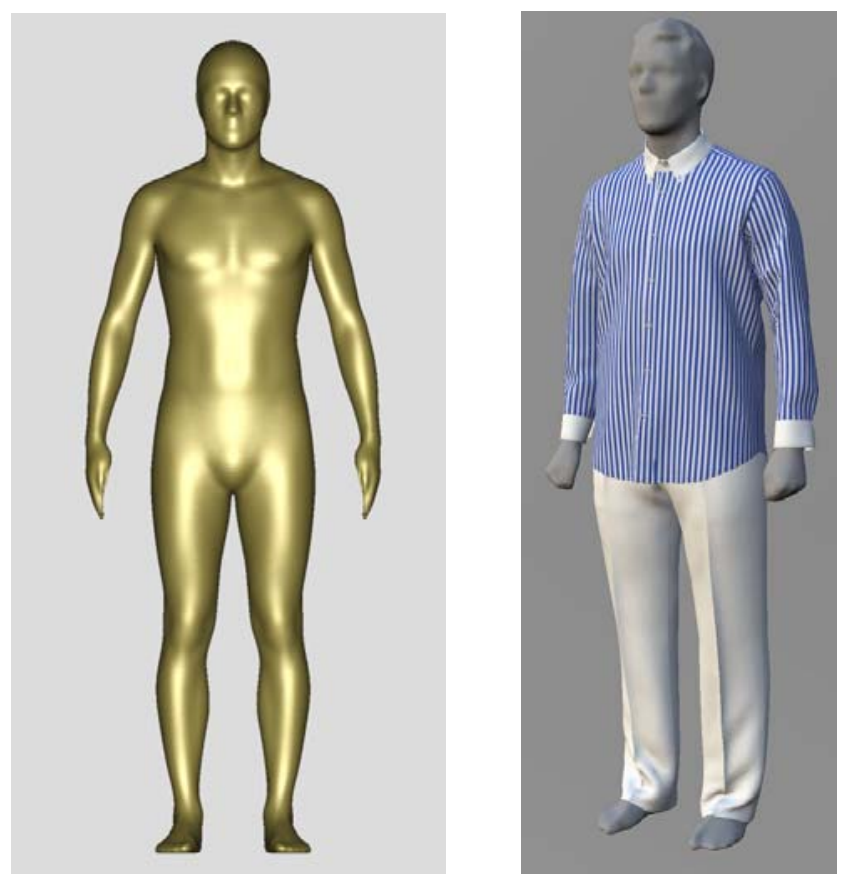

Fig. 15. Statistical scanatar and simulation of shirt with trousers.

\section{References}

1. ISO 7250:1996 Basic human body measurements for technological designs

2. ISO 8859:1989 Garment construction and anthropometric surveys - Body dimensions

3. DIN 33402:2004 Körpermaße des Menschen

4. Robert Niles (2006). Statistics Every Writer Should Know", Journalism Help, RobertNiles.com

5. Robinette, K. M., Daanen, H. A. M., Zehner, G. F. (2004). Three Dimensional Anthropometry. In Delleman, N.J., Haslegrave, C.M., Chaffin, D.B. Working Postures and Movements (pp 29-49). CRC Press LLC. 